\title{
A Self-Similar Flow behind a Magnetogasdynamic Shock Wave Generated by a Moving Piston in a Gravitating Gas with Variable Density: Isothermal Flow
}

\author{
G. Nath ${ }^{1}$ and A. K. Sinha ${ }^{2}$ \\ ${ }^{1}$ Department of Mathematics, National Institute of Technology Raipur, G. E. Road, Raipur 492010, India \\ ${ }^{2}$ Department of Mathematics, K.S. Saket P.G. College, Faizabad 224001, India \\ Correspondence should be addressed to G. Nath, gn_chaurasia_univgkp@yahoo.in
}

Received 20 April 2011; Accepted 28 July 2011

Academic Editor: Neil Sullivan

Copyright ( 2011 G. Nath and A. K. Sinha. This is an open access article distributed under the Creative Commons Attribution License, which permits unrestricted use, distribution, and reproduction in any medium, provided the original work is properly cited.

\begin{abstract}
The propagation of a cylindrical (or spherical) shock wave in an ideal gas with azimuthal magnetic field and with or without selfgravitational effects is investigated. The shock wave is driven out by a piston moving with time according to power law. The initial density and the initial magnetic field of the ambient medium are assumed to be varying and obeying power laws. Solutions are obtained, when the flow between the shock and the piston is isothermal. The gas is assumed to have infinite electrical conductivity. The shock wave moves with variable velocity, and the total energy of the wave is nonconstant. The effects of variation of the piston velocity exponent (i.e., variation of the initial density exponent), the initial magnetic field exponent, the gravitational parameter, and the Alfven-Mach number on the flow field are obtained. It is investigated that the self-gravitation reduces the effects of the magnetic field. A comparison is also made between gravitating and nongravitating cases.
\end{abstract}

\section{Introduction}

The explanation and analysis for the internal motion in stars is one of the basic problems in astrophysics. According to the observational data, the unsteady motion of large mass of the gas was followed by sudden release of energy results flare-ups in novae and supernovae. A qualitative behaviour of the gaseous mass may be discussed with the help of the equations of motion and equilibrium taking gravitational forces into account. Numerical solutions for self-similar adiabatic flows in self-gravitating gas were obtained by Sedov [1] and Carrus et al. [2], independently. Purohit [3] and Singh and Vishwakarma [4] have discussed homothermal flows behind a spherical shock wave in a self-gravitating gas using similarity method. Nath et al. [5] have studied the above problem assuming the flow to be adiabatic and selfsimilar and obtained the effects of the presence of a magnetic field. Shock wave through a variable density medium have been treated by Sedov [1], Sakurai [6], Rogers [7], Rosenau and Frankenthal [8], Nath et al. [5], Vishwakarma and Yadav [9], Nath [10], and others. Their results are more applicable to the shock formed in the deep interior of stars. Also, Vishwakarma and Singh [11] obtained the similarity solution for the flow behind a shock wave in a gravitating or nongravitating nonuniform gas with heat conduction and radiation heat flux in the case of adiabatic flow.

In aerodynamics, the analogy between the steady hypersonic flow past slender-blunted (planar or axisymmetric) power-law bodies and the one-dimensional unsteady selfsimilar flow behind a shock driven out by a piston moving with time according to a power-law is well known (see, e.g., $[1,8,10,12-19])$.

Since at high temperatures that prevail in the problems associated with the shock waves a gas is ionized, electromagnetic effects may also be significant. A complete analysis of such a problem should, therefore, consist of the study of the gas-dynamic flow and the electromagnetic field simultaneously. A detailed study towards gaining a better understanding of the interaction between gasdynamic motion of an electrically conducting medium and magnetic field within the context of hyperbolic system has been carried out by many investigators such as Korobeinikov [20], Shang 
[21], and Lock and Mestel [22]. A detailed review in the field of magnetogasdynamic flows can be seen in the paper of Shang [21]. Lock and Mestel [22] analyzed the annular selfsimilar solutions in ideal magnetogasdynamics by casting the ideal magnetogasdynamic equations to a three-dimensional autonomous system in which either the magnetic pressure or the fluid pressure vanishes.

In all of the works, mentioned above, the effect of selfgravitation is not taken into account by any of the authors in the case of isothermal flow with magnetic field.

The purpose of this study is, therefore, to obtain the selfsimilar solutions for the propagation of magnetogasdynamic cylindrical (or spherical) shock wave generated by a moving piston in a nonuniform gas with or without self-gravitational effects, in the presence of an azimuthal magnetic field, under isothermal flow condition. The media ahead and behind the shock front are assumed to be inviscid and to behave as thermally perfect gases. The counter pressure (the pressure ahead of the shock) is taken into account. The azimuthal magnetic field and the density in the ambient medium are assumed to vary and obey the power laws. The assumption of isothermal flow is physically realistic, when radiation heat transfer effects are implicitly present. As the shock propagates, the temperature behind it increases and becomes very large so that there is intense transfer of energy by radiation. This causes the temperature gradient to approach zero; that is, the dependent temperature tends to become uniform behind the shock front, and the flow becomes isothermal $[10,17,20,23-27]$. A detailed mathematical theory of one-dimensional isothermal blast waves in a magnetic field was developed by Lerche $[28,29]$. Numerical solutions for the flow field between the shock and the piston are obtained in the case of isothermal flow in Section 3. Effects of viscosity and rotation are not taken into account.

\section{Equations of Motion and Boundary Conditions}

The fundamental equations governing the unsteady and cylindrically (or spherically) symmetric isothermal flow of an electrically conducting and self-gravitating ideal gas, in the presence of an azimuthal magnetic field, may be written as (c.f. $[2,10,17,20,23-26,30])$

$$
\begin{gathered}
\frac{\partial \rho}{\partial t}+u \frac{\partial \rho}{\partial r}+\rho \frac{\partial u}{\partial r}+\frac{i u \rho}{r}=0 \\
\frac{\partial u}{\partial t}+u \frac{\partial u}{\partial r}+\frac{1}{\rho}\left[\frac{\partial p}{\partial r}+\mu h \frac{\partial h}{\partial r}+\frac{\mu h^{2}}{r}\right]+\frac{G m}{r^{i}}=0 \\
\frac{\partial h}{\partial t}+u \frac{\partial h}{\partial r}+h \frac{\partial u}{\partial r}+(i-1) \frac{h u}{r}=0 \\
\frac{\partial m}{\partial r}=2 \pi i \rho r^{i} \\
\frac{\partial T}{\partial r}=0
\end{gathered}
$$

where $r$ and $t$ are independent space and time coordinates, $u$ is the fluid velocity, $\rho$ the density, $p$ the pressure, $h$ the azimuthal magnetic field, $T$ the temperature, $\mu$ the magnetic permeability, $m$ the mass contained in a unit cylinder of radius $r$ or in a sphere of radius $r$, and the dimension of $m$ is taken as $[m]=M L^{(i-2)}$, and $i$ takes the values 2 and 1 for the respective cases of spherical and cylindrical symmetries, and $G$ the gravitational constant. In nongravitating case, (4) and the term $G m / r^{i}$ in (2) do not occur.

The electrical conductivity of the gas is assumed to be infinite. Therefore, the diffusion term from the magnetic field equation is omitted, and the electrical resistivity is ignored. Also, the effect of viscosity on the flow of the gas is assumed to be negligible.

The above system of equations should be supplemented with an equation of state. An ideal gas behaviour of the medium is assumed, so that

$$
p=\Gamma \rho T, \quad e=\frac{p}{(\gamma-1) \rho},
$$

where $\Gamma$ is the gas constant, and $\gamma$ is the ratio of specific heats.

A strong cylindrical (or spherical) shock wave is supposed to be propagating in the undisturbed ideal gas with variable density $\rho=A r^{w}$ in the presence of an azimuthal magnetic field $h=B r^{-\alpha}$, where $A, w, B$, and $\alpha$ are constant.

The flow variables immediately ahead of the shock front are

$$
\begin{gathered}
u_{1}=0, \\
\rho=\rho_{1}=A r_{s}^{w}, \\
m=m_{1}=\frac{2 \pi i A}{(i+w+1)} r_{s}^{i+w+1}, \\
h=h_{1}=B r_{s}^{-\alpha}, \\
p=p_{1}=\frac{\mu B^{2}(1-\alpha)}{2 \alpha} r_{s}^{-2 \alpha} \\
-\frac{\pi A^{2} G i}{(w+i+1)(w+1)} r_{s}^{2 w+2}+\text { constant, }
\end{gathered}
$$

where $-(i+1)<w<-1, \alpha=-(w+1), r_{s}$ is the shock radius, and the subscript " 1 " refers to the conditions immediately ahead of the shock.

The jump conditions at the magnetogasdynamic shock wave are given by the principle of conservation of mass, momentum, and energy across the shock; namely,

$$
\begin{gathered}
\rho_{1} V=\rho_{2}\left(V-u_{2}\right), \\
h_{1} V=h_{2}\left(V-u_{2}\right), \\
p_{1}+\frac{1}{2} \mu h_{1}^{2}+\rho_{1} V^{2}=p_{2}+\frac{1}{2} \mu h_{2}^{2}+\rho_{2}\left(V-u_{2}\right)^{2}, \\
e_{1}+\frac{p_{1}}{\rho_{1}}+\frac{1}{2} V^{2}+\frac{\mu h_{1}^{2}}{\rho_{1}}-\frac{q_{1}}{\rho_{1} V}=e_{2}+\frac{p_{2}}{\rho_{2}}+\frac{1}{2}\left(V-u_{2}\right)^{2} \\
+\frac{\mu h_{2}^{2}}{\rho_{2}}-\frac{q_{2}}{\rho_{1} V}, \\
m_{1}=m_{2},
\end{gathered}
$$


where the subscript " 2 " denotes the conditions immediately behind the shock front, $V\left(=d r_{s} / d t\right)$ denotes the velocity of the shock front, and " $q$ " is the radiation heat flux.

From (8), we obtain

$$
\begin{gathered}
u_{2}=(1-\beta) V, \\
\rho_{2}=\frac{\rho_{1}}{\beta}, \\
p_{2}=\left[(1-\beta)+\frac{1}{\gamma M^{2}}+\frac{M_{A}^{-2}}{2}\left(1-\frac{1}{\beta^{2}}\right)\right] \rho_{1} V^{2}, \\
m_{2}=m_{1}, \\
h_{2}=\frac{h_{1}}{\beta},
\end{gathered}
$$

where $M=\left(\rho_{1} V^{2} / \gamma p_{1}\right)^{1 / 2}$ is the shock-Mach number referred to the frozen speed of sound $\left(\gamma p_{1} / \rho_{1}\right)^{1 / 2}$, and $M_{A}=$ $\left(\rho_{1} V^{2} / \mu h_{1}^{2}\right)^{1 / 2}$ is the Alfven-Mach number. The quantity $\beta(0<\beta<1)$ is obtained by the relation

$$
\begin{aligned}
& {\left[\beta^{2}(\gamma+1)-\beta\left[\frac{2}{M^{2}}+\gamma\left(1+M_{A}^{-2}\right)-1\right]+(\gamma-2) M_{A}^{-2}\right.} \\
& \left.+\frac{\beta\left[(1-\beta)+1 / \gamma M^{2}+\left(M_{A}^{-2} / 2\right)\left(1-1 / \beta^{2}\right)\right](\gamma-1)\left(q_{2}-q_{1}\right)}{(1-\beta) p_{2} V}\right] \\
& =0 .
\end{aligned}
$$

As the shock is strong, we assume $\left(q_{2}-q_{1}\right)$ to be negligible in comparison with the product of $p_{2}$ and $V[10,17,18,23$, $27,31,32]$. Therefore, (10) reduces to

$$
\beta^{2}(\gamma+1)-\beta\left[\frac{2}{M^{2}}+\gamma\left(1+M_{A}^{-2}\right)-1\right]+(\gamma-2) M_{A}^{-2}=0 .
$$

Equation (5) together with (6) gives

$$
\frac{p}{p_{2}}=\frac{\rho}{\rho_{2}}
$$

\section{Self-Similarity Transformations}

The inner boundary of the flow field behind the shock is assumed to be an expanding surface (piston). In the frame work of self-similarity [1], the velocity $u_{p}=d r_{p} / d t$ of the piston is assumed to follow a power law which results in [10, 16-19]

$$
u_{p}=\frac{d r_{p}}{d t}=U_{0}\left(\frac{t}{t_{0}}\right)^{n}
$$

where $r_{p}$ is the radius of the piston, and $t_{0}$ denotes the time at a reference state, $U_{0}$ is the piston velocity at $t=t_{0}$, and $n$ is a constant. The consideration of the ambient pressure $p_{1}$ and the ambient magnetic field $h_{1}$ imposes a restriction on " $n$ ". Also, cylindrical and spherical geometries (i.e., for $i=1,2$ ) do not permit $n \leq-1$ for physical reasons. Thus, using (17), we obtain $-(1 /(i+1))<n<0$. For $-(1 /(i+1))<n<0$, the piston velocity suddenly rises at $t=0$ from zero to infinite velocity leading to the formation of a strong shock in the initial phase. The piston is then decelerated. Concerning the shock boundary conditions, self-similarity requires that the velocity of the shock $V=d r_{s} / d t$ is proportional to the velocity of the piston; that is,

$$
V=\frac{d r_{s}}{d t}=C U_{0}\left(\frac{t}{t_{0}}\right)^{n}
$$

where " $C$ " is a dimensionless constant. Using (14), the time and space coordinate can be changed into a dimensionless self-similarity variable $\eta$ as

$$
\eta=\frac{r}{r_{s}}=\left[\frac{(n+1) t_{0}{ }^{n}}{C U_{0}}\right]\left(\frac{r}{t^{n+1}}\right) .
$$

Evidently, $\eta=\eta_{p}=r_{p} / r_{s}$ at the piston, and $\eta=1$ at the shock. To obtain the similarity solutions, we write the unknown variables in the following form [10, 16-18]:

$$
\begin{gathered}
u=\frac{r}{t} U(\eta), \quad \rho=\rho_{1} D(\eta), \quad m=m_{1} N(\eta), \\
p=\frac{r^{2}}{t^{2}} \rho_{1} P(\eta), \quad \sqrt{\mu} h=\rho_{1}^{1 / 2} \frac{r}{t} H(\eta),
\end{gathered}
$$

where $U, D, N, P$, and $H$ are functions of $\eta$ only.

For the existence of similarity solutions, " $M$ " and $M_{A}$ should be constants; therefore,

$$
\alpha=-(w+1)=-\frac{n}{n+1} .
$$

Thus,

$M^{2}=\frac{2 n\{n(i+1)+i\} \mu B^{2}}{\left[-(2 n+1) \mu B^{2}\{n(i+1)+i\}-2 \pi G A^{2} i(n+1)^{2}\right] \gamma} M_{A}^{2}$,

where $-(1 /(i+1))<n<0$. Equation (18) shows that the solutions of the present problem can be reduced to the case in which the gas is nongravitating (i.e., the case in which $G=0$; the solution obtained by Nath [10] in the case of nonrotating medium for $i=1$ ).

Also, the total energy of the disturbance is given by

$$
E=2 \pi i \int_{r_{p}}^{r_{s}} \rho\left[\frac{1}{2} u^{2}+\frac{p}{\rho(\gamma-1)}+\frac{\mu h^{2}}{2 \rho}-\frac{G m}{r^{i-1}}\right] r^{i} d r .
$$

Applying the similarity transformations (16) in (19), we obtain

$$
E=2 \pi i A r_{s}^{[i+3 n /(n+1)]}\left(\frac{U_{0} C}{(n+1) t_{0}^{n}}\right)^{2 /(n+1)} J
$$

where $J=\int_{\eta_{p}}^{1}\left[P /(\gamma-1)+(1 / 2) D U^{2}+H^{2} / 2-\left(i(n+1)^{2} G_{0} /\right.\right.$ $\left.\left.\gamma M^{2}(i+w+1) \eta^{i+1}\right) N D\right] \eta^{i+2} d \eta$, and $G_{0}=2 \pi A^{2} G /\left[\mu B^{2}(1-\right.$ $\left.\alpha) / 2 \alpha-\pi i G A^{2} /(w+1)(w+1+i)\right]$ is the gravitational parameter. 
Hence, the total energy of the shock wave is nonconstant and varies as $r_{s}^{[i+3 n /(n+1)]}$.

Equation (12) with the aid of (16) and (9) yields a relation between $P$ and $D$ in the form

$$
P(\eta)=\frac{L D(\eta)}{\eta^{2}}
$$

where $L=\left[(1-\beta)+1 / \gamma M^{2}+\left(M_{A}^{-2} / 2\right)\left(1-1 / \beta^{2}\right)\right](n+1)^{2} \beta$.

By use of (16) and (21), (1) to (4) can be transformed and simplified to

$$
\begin{aligned}
& {[U-(n+1)] \frac{d D}{d \eta}+D \frac{d U}{d \eta}+\frac{(n+1) w D}{\eta}+\frac{(i+1) D U}{\eta}=0,} \\
& {[U-(n+1)] \frac{d U}{d \eta}+\frac{H}{D} \frac{d H}{d \eta}+\frac{L}{D \eta^{2}} \frac{d D}{d \eta}} \\
& +\frac{U(U-1)}{\eta}+\frac{2 H^{2}}{D \eta}+\frac{i(n+1)^{2} G_{0}}{\gamma M^{2}(i+w+1) \eta^{i+1}}=0 \text {, } \\
& \frac{d N}{d \eta}-\eta^{i} D(i+1+w)=0 \\
& {[U-(n+1)] \frac{d H}{d \eta}+H \frac{d U}{d \eta}+\frac{H}{\eta}\left[2 U-1+(n+1) \frac{w}{2}\right]} \\
& +\frac{H U(i-1)}{\eta}=0
\end{aligned}
$$

From (22)-(25), we obtain

$$
\frac{d D}{d \eta}=-\frac{D}{[U-(n+1)]}\left[\frac{d U}{d \eta}+\frac{(i+1) U}{\eta}+\frac{(n+1) w}{\eta}\right],
$$

$$
\frac{d H}{d \eta}=-\frac{H}{[U-(n+1)]}\left[\frac{d U}{d \eta}+\frac{2(i+1) U-2+(n+1) w}{2 \eta}\right],
$$

$$
\frac{d N}{d \eta}=\eta^{i} D(i+1+w)
$$

$$
\begin{aligned}
\frac{d U}{d \eta}=[ & H^{2} \eta^{2}\left[(i+1) U-1+(n+1) \frac{w}{2}\right] \\
& +L D[(i+1) U+(n+1) w] \\
& -\eta^{2}[U-(n+1)] \\
& \left.\times\left[U(U-1) D+2 H^{2}+\frac{i(n+1)^{2} G_{0} D}{\gamma M^{2}(i+w+1) \eta^{i}}\right]\right] / \\
& \eta\left[\{U-(n+1)\}^{2} D \eta^{2}-H^{2} \eta^{2}-L D\right] .
\end{aligned}
$$

Using the self-similarity transformations (16), the shock conditions (9) transform into

$$
\begin{gathered}
U(1)=(1-\beta)(n+1), \quad D(1)=\frac{1}{\beta}, \\
P(1)=\left[(1-\beta)+\frac{1}{\gamma M^{2}}+\frac{M_{A}^{-2}}{2}\left(1-\frac{1}{\beta^{2}}\right)\right](n+1)^{2}, \\
H(1)=\frac{(n+1)}{\beta M_{A}}, \quad N(1)=1 .
\end{gathered}
$$

The piston path coincides at $\eta_{p}=r_{p} / r_{s}$ with a particle path. Using (13) and (16), the relation

$$
U\left(\eta_{p}\right)=(n+1)
$$

can be derived. In addition to shock conditions (30), the kinematic condition (31) at the piston surface must be satisfied.

Normalizing the variables $u, p, \rho, m$, and $h$ with their respective values at the shock, we obtain

$$
\begin{array}{cc}
\frac{u}{u_{2}}=\frac{U(\eta)}{U(1)} \eta, & \frac{p}{p_{2}}=\frac{\rho}{\rho_{2}}=\beta D(\eta), \\
\frac{m}{m_{2}}=\frac{N(\eta)}{N(1)}, & \frac{h}{h_{2}}=\frac{H(\eta)}{H(1)} \eta .
\end{array}
$$

\section{Results and Discussion}

Distributions of the flow variables in the flow field behind the shock front are obtained by the numerical integration of (26)-(29) in the self-gravitating case and from (26), (27), and (29) in the nongravitating case with the boundary conditions (30) by the Runge-Kutta method of the fourth order. The expression for the shock-Mach number " $M$ " in the selfgravitating case with magnetic field $\left(M_{A}^{-2} \neq 0\right)$ is

$M^{2}=\frac{\mu B^{2}}{\left[\mu B^{2}((1-\alpha) / 2 \alpha)-\pi G A^{2} i /(i+w+1)(w+1)\right] \gamma} M_{A}^{2}$,

and in the nongravitating case with magnetic field $\left(M_{A}^{-2} \neq 0\right)$,

$$
G=0, \quad M^{2}=\frac{2 \alpha}{(1-\alpha) \gamma} M_{A}^{2},
$$

where " $\alpha$ " is the exponent in the law of the variation of initial magnetic field. Also, the expression for the shockMach number " $M$ " in the nonmagnetic self-gravitating case (i.e., $\left.M_{A}^{-2}=0\right)$ is

$$
M^{2}=\frac{n[n+(n+1) i](n+1)^{-2 /(n+1)}}{i \gamma \pi A G}\left(\frac{C U_{0}}{t_{0}^{n}}\right)^{2 /(n+1)},
$$

and in the nongravitating and nonmagnetic case

$$
G=0, \quad M^{2}=\infty \text {. }
$$


TABLE 1: Variation of the density ratio $\beta\left(=\rho_{1} / \rho_{2}\right)$ across the shock front and the position of the piston surface $\eta_{p}$ for different values of $M_{A}^{-2}$, $n$, and $G_{0}$ with $\gamma=5 / 3, i=2$.

\begin{tabular}{|c|c|c|c|c|c|c|c|c|}
\hline \multirow{4}{*}{$\begin{array}{l}M_{A}^{-2} \\
0.0\end{array}$} & \multirow{4}{*}{$\begin{array}{c}n \\
-1 / 4\end{array}$} & \multirow{2}{*}{\multicolumn{2}{|c|}{$w=-1 /(n+1)$}} & \multicolumn{3}{|c|}{ Gravitating case } & \multicolumn{2}{|c|}{ Nongravitating case } \\
\hline & & & & \multirow{3}{*}{$\frac{\beta(M=10)}{0.2575}$} & \multicolumn{2}{|c|}{ Position of the piston $\eta_{p}$} & \multirow{4}{*}{$\begin{array}{c}\beta(M=\infty) \\
0.2500 \\
0.2500\end{array}$} & \multirow{4}{*}{$\begin{array}{c}\eta_{p} \\
0.786644 \\
0.826576\end{array}$} \\
\hline & & \multirow{2}{*}{\multicolumn{2}{|c|}{$-4 / 3$}} & & $G_{0}=10$ & $G_{0}=100$ & & \\
\hline & & & & & 0.792961 & 0.858979 & & \\
\hline & $-1 / 6$ & \multicolumn{2}{|c|}{$-6 / 5$} & 0.2575 & 0.828341 & 0.871949 & & \\
\hline \multirow{3}{*}{$M_{A}^{-2}$} & \multirow{3}{*}{$n$} & \multirow{3}{*}{\multicolumn{2}{|c|}{$w$}} & \multirow{3}{*}{$\beta$} & \multicolumn{4}{|c|}{ Position of the piston $\eta_{p}$} \\
\hline & & & & & \multicolumn{2}{|c|}{ Gravitating case } & \multirow{2}{*}{\multicolumn{2}{|c|}{ Nongravitating case }} \\
\hline & & & & & $G_{0}=10$ & $G_{0}=100$ & & \\
\hline \multirow{2}{*}{0.01} & $-1 / 4$ & $1 / 3$ & $-4 / 3$ & 0.273323 & 0.762936 & 0.850165 & \multicolumn{2}{|c|}{0.74148} \\
\hline & $-1 / 6$ & $1 / 5$ & $-6 / 5$ & 0.285626 & 0.810785 & 0.888747 & \multicolumn{2}{|c|}{0.78455} \\
\hline \multirow{2}{*}{0.02} & $-1 / 4$ & $1 / 3$ & $-4 / 3$ & 0.295947 & 0.753016 & 0.8622504 & \multicolumn{2}{|c|}{0.705453} \\
\hline & $-1 / 6$ & $1 / 5$ & $-6 / 5$ & 0.320305 & 0.799756 & 0.898664 & \multicolumn{2}{|c|}{0.747313} \\
\hline \multirow{2}{*}{0.05} & $-1 / 4$ & $1 / 3$ & $-4 / 3$ & 0.36106 & 0.726403 & 0.870199 & \multicolumn{2}{|c|}{0.606753} \\
\hline & $-1 / 6$ & $1 / 5$ & $-6 / 5$ & 0.421092 & 0.777162 & 0.904818 & \multicolumn{2}{|c|}{0.63214} \\
\hline
\end{tabular}

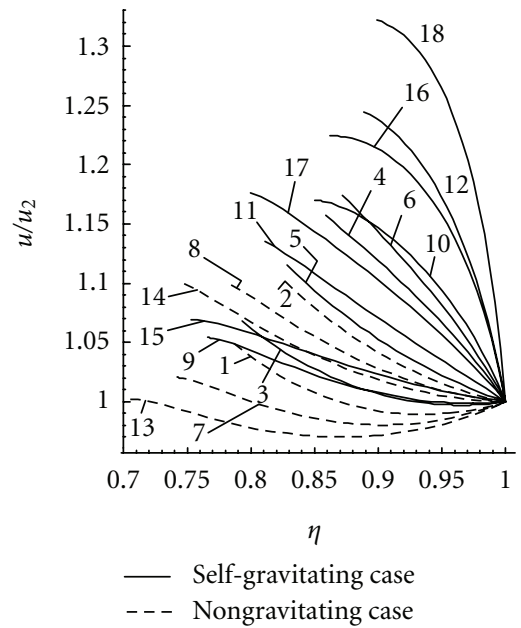

(a)

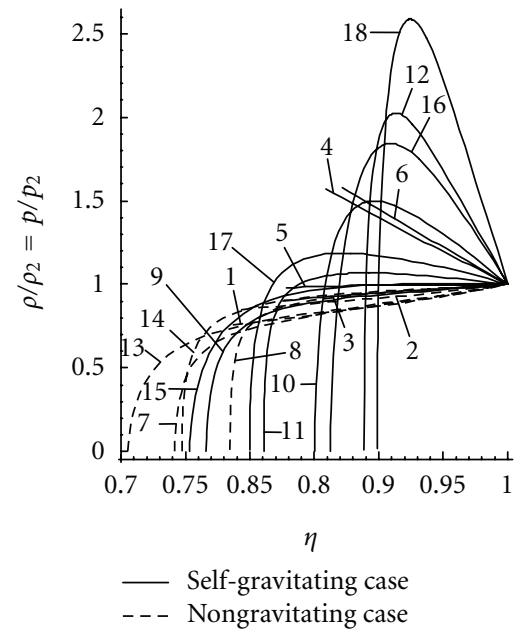

(b)

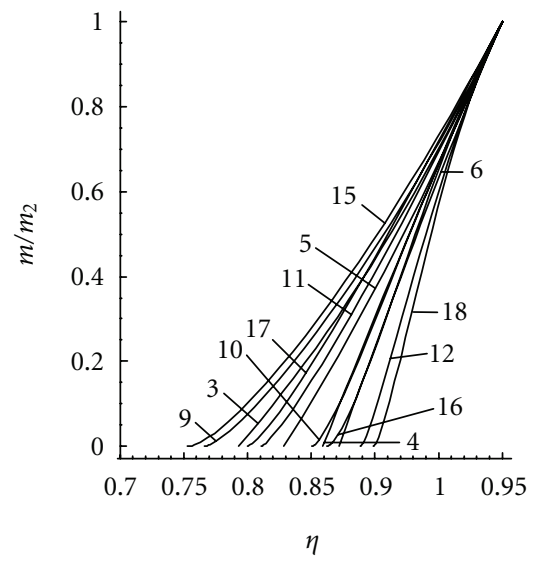

(c)

FIgURE 1: Variation of the reduced flow variables in the region behind the shock front. (a) The fluid velocity $u / u_{2}$. (b) The density (the pressure) $\left(\rho / \rho_{2}\right)\left(=p / p_{2}\right)$. (c) The mass $m / m_{2}$. (see Table 2 ).

For the purpose of numerical integration, the values of the constant parameters are taken to be $[8,10,33] \gamma=5 / 3$, $i=2, G_{0}=0,10,100, M_{A}^{-2}=0.0,0.01,0.02,0.05, n=-1 / 4$, $-1 / 6$. For fully ionized gas $\gamma=5 / 3$, and; therefore, it is applicable to stellar medium. Rosenau and Frankenthal [8] have shown that the effects of magnetic field on the flow field behind the shock are significant when $M_{A}^{-2} \geq 0.01$; therefore, the above values of $M_{A}^{-2}$ are taken for calculations in the present problem. The value $M_{A}^{-2}=0$ corresponds to the nonmagnetic case. The value $G_{0}=0$ corresponds to the solution in nongravitating case, the solution obtained by Nath [10] in non-rotating case when $\alpha=1 / 2$, $i=1$.

Table 1 shows the variation of density ratio $\beta\left(=\rho_{1} / \rho_{2}\right)$ across the shock front and the position of the piston $\eta_{p}$ for different values $M_{A}^{-2}$ with $\gamma=5 / 3, G_{0}=0,10,100$, $n=-1 / 4,-1 / 6$ in both the gravitating and nongravitating cases. Also, Table 1 shows that the distance of the piston from the shock front is less in the case of gravitating medium in comparison with that in the case of nongravitating medium. Physically, it means that the gas behind the shock is compressed in gravitating medium; that is, the shock strength is increased in gravitating medium.

Figures 1 (a) to 1 (c) and 2 show the variation of the flow variables $u / u_{2}, \rho / \rho_{2}=p / p_{2}, m / m_{2}$, and $h / h_{2}$, with $\eta$ at various values of the parameters $M_{A}^{-2}, G_{0}$, and $n$.

Figure 1 (a) shows that the reduced fluid velocity $u / u_{2}$ increases from the shock front to the piston whereas it decreases when $M_{A}^{-2}=0, n=-1 / 4$ and $M_{A}^{-2}=0.01$ or 0.02 , $n=-1 / 4$ or $-1 / 6$ in the case of nongravitating medium and $M_{A}^{-2}=0.01$ or $0.02, n=-1 / 4$ or $-1 / 6$ in the case of gravitating medium for $G_{0}=10$.

Figure 1 (c) shows that the reduced mass $\mathrm{m} / \mathrm{m}_{2}$ decreases from the shock front to the piston, and Figure 2 shows that the reduced azimuthal magnetic field $h / h_{2}$ increases from the shock front to the piston. 
TABLE 2

(1) $M_{A}^{-2}=0, n=-1 / 4, G_{0}=0$
(2) $M_{A}^{-2}=0, n=-1 / 6, G_{0}=0$
(3) $M_{A}^{-2}=0, n=-1 / 4, G_{0}=10$
(4) $M_{A}^{-2}=0, n=-1 / 6, G_{0}=10$
(5) $M_{A}^{-2}=0, n=-1 / 4, G_{0}=100$
(6) $M_{A}^{-2}=0, n=-1 / 6, G_{0}=100$
(7) $M_{A}^{-2}=0.01, n=-1 / 4, G_{0}=0$
(8) $M_{A}^{-2}=0.01, n=-1 / 6, G_{0}=0$
(9) $M_{A}^{-2}=0.01, n=-1 / 4, G_{0}=10$
(10) $M_{A}^{-2}=0.01, n=-1 / 6, G_{0}=10$
(11) $M_{A}^{-2}=0.01, n=-1 / 4, G_{0}=100$
(12) $M_{A}^{-2}=0.01, n=-1 / 6, G_{0}=100$
(13) $M_{A}^{-2}=0.02, n=-1 / 4, G_{0}=0$
(14) $M_{A}^{-2}=0.02, n=-1 / 6, G_{0}=0$
(15) $M_{A}^{-2}=0.02, n=-1 / 4, G_{0}=10$
(16) $M_{A}^{-2}=0.02, n=-1 / 6, G_{0}=10$
(17) $M_{A}^{-2}=0.02, n=-1 / 4, G_{0}=100$
(18) $M_{A}^{-2}=0.02, n=-1 / 6, G_{0}=100$

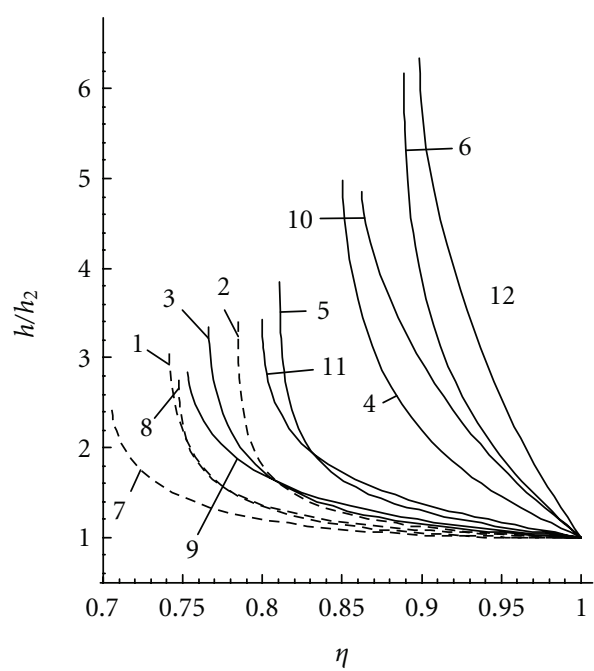

_ Self-gravitating case

FIGURE 2: Variation of the reduced azimuthal magnetic field $h / h_{2}$ in the region behind the shock front. (see Table 3 ).

Figure 1(b) shows that the reduced density (pressure) $\left(\rho / \rho_{2}\right)\left(=p / p_{2}\right)$ decreases; but it increases in the cases when (i) $n=-1 / 6, M_{A}^{-2} \neq 0, G_{0} \neq 0$ and (ii) $n=-1 / 6, M_{A}^{-2}=0$, $G_{0} \neq 0$ and starts to decrease after attaining a maximum in the cases (i) and the formation of maxima is absent in the cases (ii). As can be seen from (26) for " $D$," there is a singularity at the piston where $U\left(\eta_{p}\right)=(n+1)$ because this equation becomes singular there. This singularity is removable, and a finite solution for " $D$ " is obtained as shown in Figure 1(b) (see curves 1-6) in nonmagnetic case $\left(M_{A}^{-2}=\right.$ 0 ). In the magnetic case in Figure 1(b), the curves 7-18 show that the singularity is nonremovable, and the derivative of density tends to negative infinity.
TABLE 3

\begin{tabular}{l} 
(1) $M_{A}^{-2}=0.01, n=-1 / 4, G_{0}=0$ \\
(2) $M_{A}^{-2}=0.01, n=-1 / 6, G_{0}=0$ \\
(3) $M_{A}^{-2}=0.01, n=-1 / 4, G_{0}=10$ \\
(4) $M_{A}^{-2}=0.01, n=-1 / 6, G_{0}=10$ \\
(5) $M_{A}^{-2}=0.01, n=-1 / 4, G_{0}=100$ \\
(6) $M_{A}^{-2}=0.01, n=-1 / 6, G_{0}=100$ \\
(7) $M_{A}^{-2}=0.02, n=-1 / 4, G_{0}=0$ \\
(8) $M_{A}^{-2}=0.02, n=-1 / 6, G_{0}=0$ \\
(9) $M_{A}^{-2}=0.02, n=-1 / 4, G_{0}=10$ \\
(10) $M_{A}^{-2}=0.02, n=-1 / 6, G_{0}=10$ \\
(11) $M_{A}^{-2}=0.02, n=-1 / 4, G_{0}=100$ \\
(12) $M_{A}^{-2}=0.02, n=-1 / 6, G_{0}=100$ \\
\hline
\end{tabular}

This singularity can be physically interpreted as follows $[10,16,18]$ : the path of the piston diverges from the path of the particle immediately ahead rarifying the gas.

The piston position $\eta_{p}$ at which $U=(n+1)$, is obtained after numerical integration of (26) to (29) in the self-gravitating case and from (26), (27), and (29) in the nongravitating case with the boundary conditions (30) and is tabulated in Table 1 for different values of $M_{A}^{-2}, n$, and $G_{0}$. The piston position $\eta_{p}$ is related with the velocity ratio of shock and piston, from (22), (23), and (24) as follows:

$$
\eta_{p}=\frac{1}{C}=\left(\frac{V}{u_{p}}\right)^{-1} .
$$

From Table 1 and Figures $1(\mathrm{a})-1(\mathrm{c})$ and 2 , it is found that the effects of an increase in the value of $M_{A}^{-2}$ (i.e., the effect of an increase in the strength of ambient magnetic field) are the following.

(i) To decrease $\eta_{p}$ when $G_{0}=0$ or 10 (i.e., to increase the distance of the piston from the shock front), whereas to increase it when $G_{0}=100$ (i.e., to decrease the distance of the piston from the shock front). Physically it means that the gas behind the shock is less compressed when $G_{0}=0$ or 10 , whereas it is more compressed when $G_{0}=100$, that is, to decrease the shock strength in the case when $G_{0}=0$ or 10 and to increase it when $G_{0}=100$ (see Table 1). Thus, the effect of an increase in the strength of initial magnetic field is reduced by an increase in the strength of selfgravitation.

(ii) To increase the value of $\beta$ (i.e., to decrease the shock strength) (see Table 1).

(iii) To increase the reduced fluid velocity $u / u_{2}$ and the reduced azimuthal magnetic field $h / h_{2}$ in the case of self-gravitating medium but to decrease these flow variables in the case of nongravitating medium (see Figures 1(a) and 2).

(iv) To increase the reduced mass $\mathrm{m} / \mathrm{m}_{2}$ but to decrease it when $n=-1 / 6$ and $G_{0}=10$ or 100 (see Figure 1(c)).

(v) To increase the reduced density (pressure) (see Figure 1(b)). 
The effects of an increase in the value of the piston velocity exponent $n$ or the exponent $w$ for the variation of the initial density (i.e., the effects of the decrease in the value of the exponent $\alpha$ for the variation of the initial magnetic field) of the ambient medium are the following.

(i) To increase $\eta_{p}$, that is, to decrease the distance of the piston from the shock front. Physically, it means that the gas behind the shock is more compressed; that is, the shock strength is increased (see Table 1).

(ii) To increase the value of $\beta$, when $M_{A}^{-2} \neq 0$ (i.e., to decrease the shock strength), which is same as in (i) (see Table 1).

(iii) To increase the reduced fluid velocity $u / u_{2}$ and the reduced azimuthal magnetic field $h / h_{2}$ (see Figures 1 (a) and 2).

(iv) To decrease the reduced mass $\mathrm{m} / \mathrm{m}_{2}$ (see Figure 1(c)).

(v) To increase the reduced density (pressure) in general (see Figure 1(b)).

Effects of an increase in the value of the gravitational parameter $G_{0}$ are

(i) to increase $\eta_{p}$, that is, to decrease the distance of the piston from the shock front (see Table 1),

(ii) to increase the flow variables the reduced fluid velocity $u / u_{2}$, the reduced density (pressure) $\left(\rho / \rho_{2}\right)(=$ $\left.p / p_{2}\right)$, and the reduced azimuthal magnetic field $h / h_{2}$ (see Figures 1(a), 1(b) and 2),

(iii) to decrease the reduced mass $\mathrm{m} / \mathrm{m}_{2}$ (see Figure 1(c)).

\section{Acknowledgment}

The authors are grateful to Dr. J. P. Vishwakarma, Professor of mathematics, DDU Gorakhpur University Gorakhpur273009, India, for many useful discussions.

\section{References}

[1] L. I. Sedov, Similarity and Dimensional Methods in Mechanics, Academic Press, New York, NY, USA, 1959.

[2] P. Carrus, P. Fox, F. Hass, and Z. Kopal, "The propagation of shock waves in a steller model with continuous density distribution," The Astrophysical Journal, vol. 113, pp. 496-518, 1951.

[3] S. C. Purohit, "Self-similar homothermal flow of selfgravitating gas behind shock wave," Journal of the Physical Society of Japan, vol. 36, no. 1, pp. 288-292, 1974.

[4] J. B. Singh and P. R. Vishwakarma, "Self-similar solutions in the theory of flare-ups in novae, I," Astrophysics and Space Science, vol. 95, no. 1, pp. 99-104, 1983.

[5] O. Nath, S. Ojha, and H. S. Takhar, "A study of stellar point explosion in a self-gravitating radiative magnetohydrodynamic medium," Astrophysics and Space Science, vol. 183, no. 1, pp. 135-145, 1991.

[6] A. Sakurai, "Propagation of spherical shock waves in stars," Journal of Fluid Mechanics, vol. 1, pp. 436-453, 1956.

[7] M. H. Rogers, "Analytic solutions for blast wave problem with an atmosphere of varying density," Astrophysical Journal, vol. 125, pp. 478-493, 1957.
[8] P. Rosenau and S. Frankenthal, "Equatorial propagation of axisymmetric magnetohydrodynamic shocks," Physics of Fluids, vol. 19, no. 12, pp. 1889-1899, 1976.

[9] J. P. Vishwakarma and A. K. Yadav, "Self-similar analytical solutions for blast waves in inhomogeneous atmospheres with frozen-in-magnetic field," European Physical Journal B, vol. 34, no. 2, pp. 247-253, 2003.

[10] G. Nath, "Magnetogasdynamic shock wave generated by a moving piston in a rotational axisymmetric isothermal flow of perfect gas with variable density," Advances in Space Research, vol. 37, no. 9, pp. 1463-1471, 2011.

[11] J. P. Vishwakarma and A. K. Singh, "A self-similar flow behind a shock wave in a gravitating or non-gravitating gas with heat conduction and radiation heat-flux," Journal of Astrophysics and Astronomy, vol. 30, no. 1, pp. 53-69, 2009.

[12] S. S. Grigobian, "The Cauchy problem and piston problem for one-dimensional unsteady motions of a gas: self-similar motions," Journal of Applied Mathematics and Mechanics, vol. 22, no. 2, pp. 179-187, 1958.

[13] N. N. Kochina and N. S. Mel'nikova, "On unsteady motion of a gas forced out by a piston with counter pressure neglected," Journal of Applied Mathematics and Mechanics, vol. 22, no. 4, pp. 444-451, 1958.

[14] K. C. Wang, "The piston problem with thermal radiation," Journal of Fluid Mechanics, vol. 20, pp. 447-455, 1964.

[15] J. B. Helliwell, "Self- similar piston problem with radiative heat transfer," Journal of Fluid Mechanics, vol. 37, no. 3, pp. 497-512, 1969.

[16] H. Steiner and T. Hirschler, "A self-similar solution of a shock propagation in a dusty gas," European Journal of Mechanics B/Fluids, vol. 21, no. 3, pp. 371-380, 2002.

[17] G. Nath, "Shock waves generated by a piston moving in a nonideal gas in the presence of a magnetic field: isothermal flow," South East Asian Journal of Mathematics and Mathematical Sciences, vol. 5, pp. 69-83, 2007.

[18] J. P. Vishwakarma and G. Nath, "A self- similar solution of a shock propagation in a mixture of a non-ideal gas and small solid particles," Meccanica, vol. 44, no. 3, pp. 239-254, 2009.

[19] J. P. Vishwakarma and G. Nath, "Propagation of a cylindrical shock wave in a rotating dusty gas with heat conduction and radiation heat flux," Physica Scripta, vol. 81, no. 4, Article ID 045401, 2010.

[20] V. P. Korobeinikov, Problems in the Theory of Point Explosion in Gases, Proceedings of the Steklov Institute of Mathematics Series No. 119, American Mathematical Society, 1976.

[21] J. S. Shang, "Recent research in magneto-aerodynamics," Progress in Aerospace Sciences, vol. 37, no. 1, pp. 1-20, 2001.

[22] R. M. Lock and A. J. Mestel, "Annular self-similar solutions in ideal magnetogasdynamics," Journal of Plasma Physics, vol. 74, no. 4, pp. 531-554, 2008.

[23] D. D. Laumbach and R. F. Probstein, "Self-similar strong shocks with radiation in a decreasing exponential atmosphere," Physics of Fluids, vol. 13, no. 5, pp. 1178-1183, 1970.

[24] P. L. Sachdev and S. Ashraf, "Converging spherical and cylindrical shocks with zero temperature gradient in the rear flow field," Journal of Applied Mathematics and Physics, vol. 22, no. 6, pp. 1095-1102, 1971.

[25] S. Ashraf and Z. Ahmad, "Approximate analytic solution of a strong shock with radiation near the surface of the star," Indian Journal of Pure and Applied Mathematics, vol. 6, pp. 1090-1098, 1975. 
[26] T. A. Zhuravskaya and V. A. Levin, "The propagation of converging and diverging shock waves under intense heatexchange conditions," Journal of Applied Mathematics and Mechanics, vol. 60, no. 5, pp. 745-752, 1996.

[27] G. Nath, "Propagation of a strong cylindrical shock wave in a rotational axisymmetric dusty gas with exponentially varying density," Research in Astronomy and Astrophysics, vol. 10, no. 5, pp. 445-460, 2010.

[28] I. Lerche, "Mathematical theory of one-dimensional isothermal blast waves in a magnetic field," Australian Journal of Physics, vol. 32, pp. 491-502, 1979.

[29] I. Lerche, "Mathematical theory of one-dimensional cylindrical isothermal blast waves in a magnetic field," Australian Journal of Physics, vol. 34, pp. 279-301, 1981.

[30] G. B. Whitham, "On the propagation of shock waves through regions of non-uniform area or flow," Journal of Fluid Mechanics, vol. 4, pp. 337-360, 1958.

[31] J. P. Vishwakarma and G. Nath, "Similarity solutions for unsteady flow behind an exponential shock in a dusty gas," Physica Scripta, vol. 74, no. 4, article 015, pp. 493-498, 2006.

[32] J. P. Vishwakarma and G. Nath, "Similarity solutions for the flow behind an exponential shock in a non-ideal gas," Meccanica, vol. 42, no. 4, pp. 331-339, 2007.

[33] J. P. Vishwakarma, A. K. Maurya, and K. K. Singh, "Self-similar adiabatic flow headed by a magnetogasdynamic cylindrical shock wave in a rotating non-ideal gas," Geophysical and Astrophysical Fluid Dynamics, vol. 101, no. 2, pp. 155-168, 2007. 

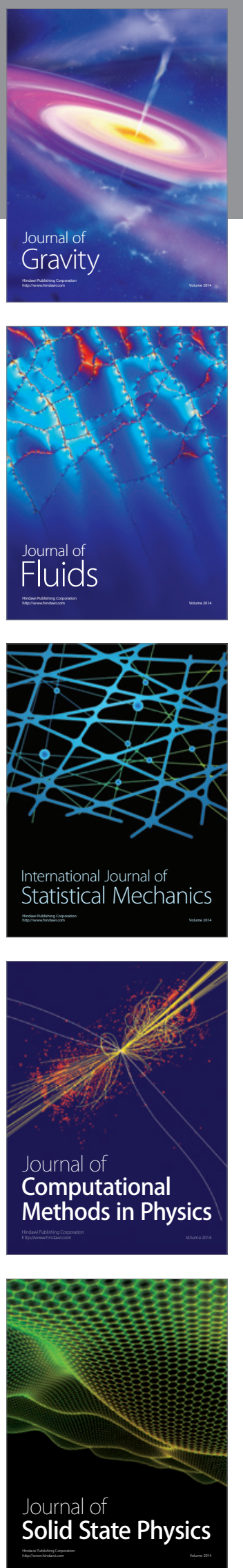

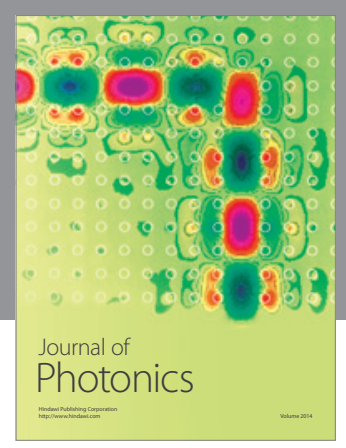

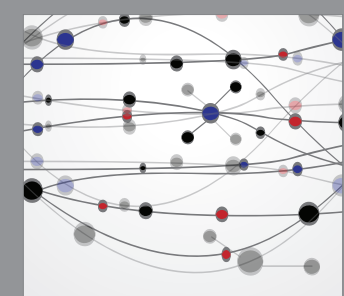

The Scientific World Journal
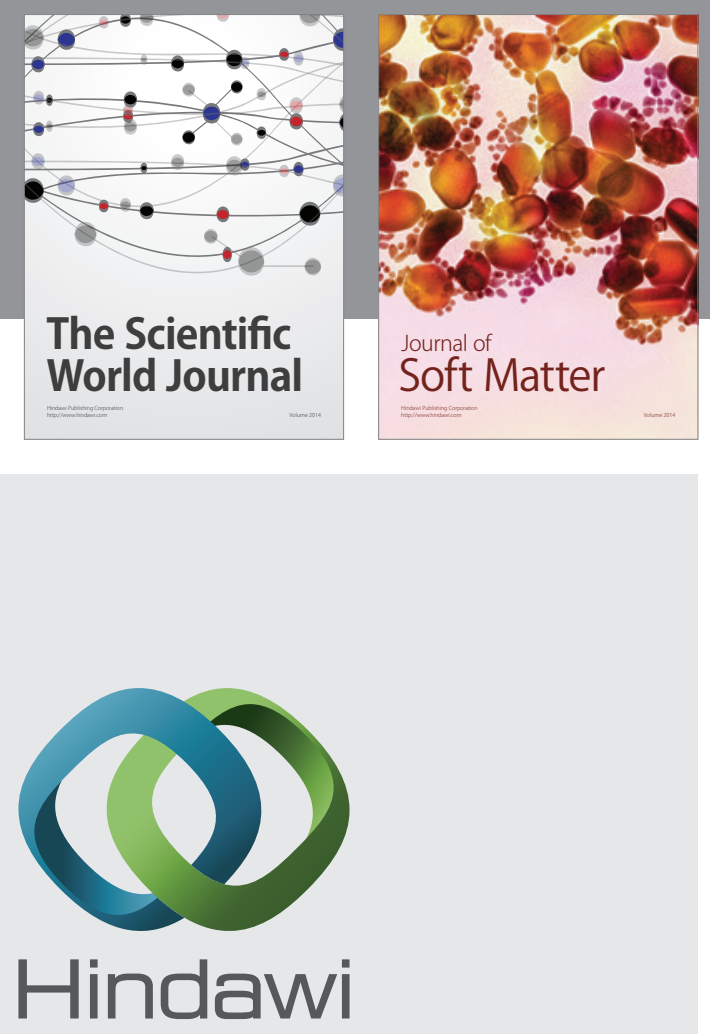

Submit your manuscripts at

http://www.hindawi.com
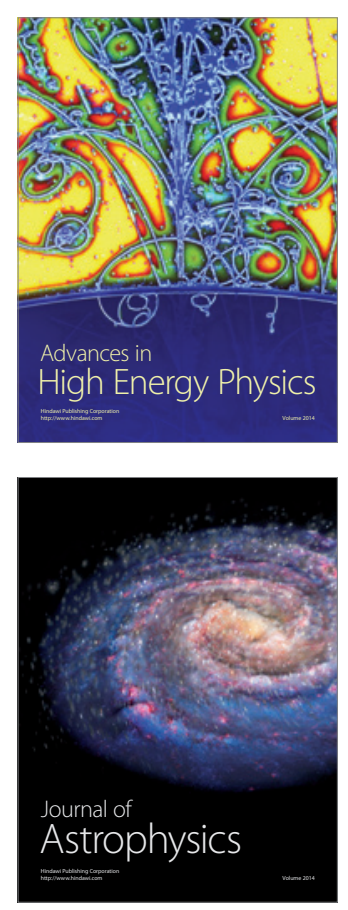
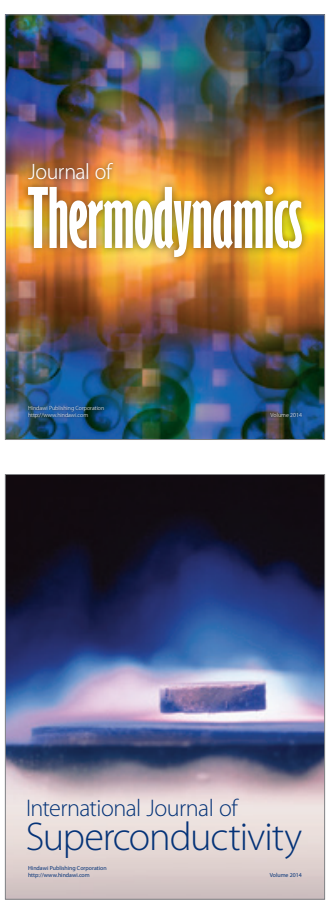
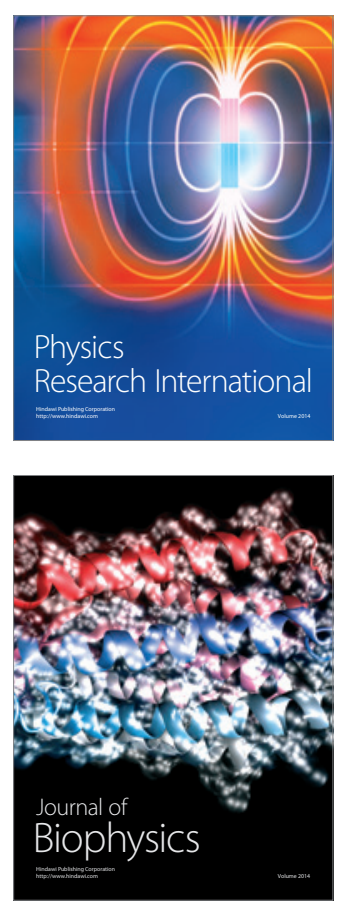
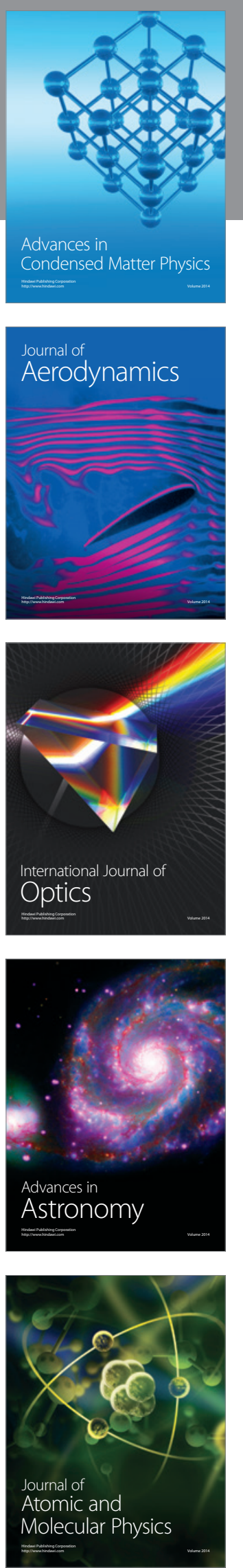